Open Access

\title{
Estrogen stimulates SREBP2 expression in hepatic cell lines via an estrogen response element in the SREBP2 promoter
}

Ye Meng ${ }^{*}$ (i) and Lu Zong

\author{
* Correspondence: mengye@ustc. \\ edu.cn \\ The First Affiliated Hospital of USTC, \\ Division of Life Sciences and \\ Medicine, University of Science and \\ Technology of China, Hefei, Anhui \\ 230001, People's Republic of China
}

\begin{abstract}
Objective: Hypoestrogenism in women is strongly associated with menopause and it can lead to lipid disorder, which predisposes people to premature cardiovascular disease. However, the mechanism of lipid disorder remains unclear. Sterol regulatory element-binding protein 2 (SREBP2) is the key transcription factor regulating cholesterol metabolism. We hypothesize that estrogen regulates SREBP2 transcription through an estrogen response element (ERE) in the SREBP2 promoter region.

Methods: Human hepatoblastoma cells (HepG2) were treated with dose-dependent concentrations of estradiol $\left(E_{2}\right)$ for $24 \mathrm{~h}$. Then, SREBP2 expression was determined via real-time PCR and immunofluorescence. The expressions of the SREBP2 downstream target genes HMGCR and LDLR were determined via real-time PCR. Lipid secretion in the culture media of HepG2 cells was measured using ELISA. Through bioinformatics analysis, we identified high-scoring ERE-like sequences in the SREBP2 gene promoter. Chromatin immunoprecipitation analysis was used to confirm the ERE. DNA fragments of the putative or mutated ERE-like sequence were synthesized and ligated into pGL3basic plasmid to construct the SREBP2 promoter luciferase reporter systems. SREBP2Luciferase (SREBP2-LUC), SREBP2-Mutation (SREBP2-Mut) and the blank control were transfected into hepatic cell lines. Luciferase activities were measured using the dualluciferase reporter assay system. Chromatin immunoprecipitation analysis and the luciferase reporter assay were repeated in human hepatoma cells ( $\mathrm{HuH}-7)$.
\end{abstract}

Results: We found that $E_{2}$ dose-dependently increased the expression of SREBP2 in HepG2 cells and that the increased levels were blocked when treated with an estrogen receptor-alpha antagonist. Additionally, $E_{2}$ increased both HMGCR and LDLR expression and lipid secretion in HepG2 cells. Notably, we identified a functional ERE in the SREBP2 gene promoter, to which $\mathrm{E}_{2}$ could specifically bind and induce transcription.

Conclusions: An ERE was identified in the SREBP2 gene promoter. It mediates the regulation of SREBP2 expression by estrogen in hepatocytes. This study provides a mechanism to link cardiovascular disease with estrogen.

Keywords: Sterol regulatory element-binding protein 2, Estradiol, Transcription regulation, Lipid metabolism

\section{Introduction}

Effective methods to prevent cardiovascular diseases are essential, since they are major causes of morbidity and mortality around the world [1]. Examples include coronary

(c) The Author(s). 2019 Open Access This article is distributed under the terms of the Creative Commons Attribution 4.0 International License (http://creativecommons.org/licenses/by/4.0/), which permits unrestricted use, distribution, and reproduction in any medium, provided you give appropriate credit to the original author(s) and the source, provide a link to the Creative Commons license, and indicate if changes were made. The Creative Commons Public Domain Dedication waiver (http://creativecommons.org/ publicdomain/zero/1.0/) applies to the data made available in this article, unless otherwise stated. 
artery disease, congestive heart failure, peripheral vascular disease, cerebrovascular disease and left ventricular hypertrophy [2].

A large number of epidemiological studies have shown that determinants of cardiovascular disease include behavior, environmental factors and heredity factors [3]. Risk factors of cardiovascular disease include cholesterol level, body mass index (BMI), blood pressure and fasting plasma glucose [4]. Dyslipidemia is an important underlying risk factor, especially in terms of elevated total cholesterol (TC) and low-density lipoprotein cholesterol (LDL-C) levels $[5,6]$. LDL-C is the current primary therapeutic target, and lowering its levels, most commonly by treatment with statins, is the current prevention approach. In addition, decreased total cholesterol and triglycerides (TG) are emerging as reliable therapeutic targets of cardiovascular disease [7, 8].

The incidence of cardiovascular disease increases sharply in females after menopause. Studies have shown that this can be mainly attributed to lipid disorder, vascular stability destruction and blood pressure increase, which result from a series of pathological changes caused by decreased estrogen levels [9-11].

As an important steroid hormone, estrogen mainly regulates estrogen-sensitive genes via the classical pathway: estrogen receptor $\alpha(E R \alpha)$ binds to estrogen response elements (EREs) to regulate gene transcription [12]. Estrogen is involved in the functional regulation of multiple organs and systems, and its role in the progression of cardiovascular disease has attracted much attention in recent years. Studies have indicated that estrogen can regulate lipid homeostasis in the adipose tissue, liver and brain, as well as prevent metabolic dyslipidemia [13]. Furthermore, clinical evidence demonstrates that estrogen replacement therapy can reduce the risk of cardiovascular disease in postmenopausal women by improving lipid metabolism $[14,15]$. Further studies are needed to identify the mechanisms by which estrogen regulates lipid metabolism and delays the development of cardiovascular disease in postmenopausal women.

Sterol regulatory element-binding proteins (SREBPs) are a family of key nuclear transcription factors that can regulate lipid metabolism by controlling the expression of a series of enzymes required for the synthesis of endogenous cholesterol, triacylglycerol, fatty acid and phospholipid [16]. Nuclear SREBPs activate lipid metabolism-related enzymes by binding to specific sterol regulatory elements (SREs) in the promoters of target genes [17]. There are three isoforms: SREBP1a, SREBP1c and SREBP2. Each plays a different role in lipid synthesis. SREBP1a is the master regulator of lipogenesis, especially in fatty acid and triglyceride biosynthesis. SREBP1c regulates fatty acid synthesis and insulin-induced glucose homeostasis. SREBP2 is a crucial factor for (and relatively specific to) cholesterol synthesis and plays an important role in the self-feedback control of intracellular cholesterol $[18,19]$.

The aim of this investigation was to evaluate whether SREBP2 is regulated by estrogen and to further understand the regulatory pathway. Lipid metabolism mainly occurs in the liver, so we focused on hepatocytes to study this mechanism.

\section{Materials and methods}

\section{Cell culture and ELISA assays}

Human hepatoblastoma (HepG2) and hepatoma (HuH-7) cell lines were purchased from the Cell Resource Center of the Shanghai Institutes for Biological Sciences of the 
Chinese Academy of Sciences. These cells were cultured at $37^{\circ} \mathrm{C}$ in $95 \% \mathrm{CO}_{2}$ in highglucose phenol red Dulbecco's modified Eagle medium (DMEM; Gibco-BRL) supplemented with $10 \%$ fetal bovine serum (FBS; Gibco-BRL) and $100 \mathrm{U} / \mathrm{ml}$ streptomycin and penicillin (Gibco-BRL). After the cells were $40-50 \%$ confluent, the medium was replaced with phenol red-free DMEM (Gibco-BRL) supplemented with $1 \%$ charcoalstripped FBS (Gibco-BRL). 17 $\beta$-estradiol ( $E_{2} ; 7.14 \mathrm{nmol} / \mathrm{l}$; Sigma-Aldrich), which is the most active form of estrogen [20], was added to the culture medium at gradient concentrations $\left(0,10^{-9}, 10^{-7}\right.$ and $\left.10^{-5} \mathrm{~mol} / \mathrm{l}\right)$ for $24 \mathrm{~h}$.

The concentrations of TC, TG, LDL-C and high-density lipoprotein cholesterol (HDL-C) in the culture media of HepG2 cells were measured using an ELISA kit $(R \& D)$ according to the manufacturer's instructions.

\section{RT-PCR and quantitative real-time PCR analysis}

Total RNA was extracted from scraped cells using Trizol reagent (Takara) and reverse transcription was performed using the protocol of the PrimeScript RT reagent Kit (Takara). PCR was carried out in a thermal cycler (PTC-200 DNA Engine; MJ Research). The RT-PCR product was visualized in a $1 \%$ agarose gel.

Real-time PCR was performed using an Applied Biosystems 7900 Fast Real-Time PCR System (Applied Biosystems). After normalizing to glyceraldehyde-3-phosphate dehydrogenase (GAPDH), the data were analyzed using the comparative threshold cycle method. Changes after treatment were noted as fold differences from untreated control values. The primer sequences for SREBP2 were 5'-GTCGGGTGTCATGGGCGGTG3' and 5'-CTCGCCGCTGTCGTCGATCG-3'; for 3-hydroxy-3-methylglutaryl-CoA reductase (HMGCR) were 5'-TGAGGGCTCCTTCCGCTCCG-3' and 5'-ACTAGA GGCCACCGAACCCCG-3'; for low density lipoprotein receptor (LDLR) were 5' TACCCCTCGAGACAGATGGT-3' and 5'-CACTGTCCGAAGCCTGTTCT-3'; and

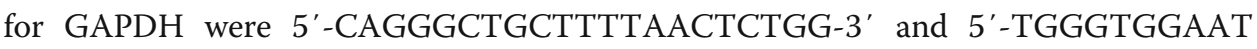
CATATTGGAACA-3'.

\section{Immunofluorescence staining}

Approximately $10^{4}$ HepG2 cells were grown on coverslips and treated with dimethyl sulfoxide (DMSO), $\mathrm{E}_{2}\left(10^{-7} \mathrm{~mol} / \mathrm{l}\right)$, or $\mathrm{E}_{2}\left(10^{-7} \mathrm{~mol} / \mathrm{l}\right)+\mathrm{ICI}$ (ICI 182,780, 7 $\alpha$-[9-(4,4,5,5, 5-pentafluoro-pentylsulphinyl)nonyl]oestra-1,3,5(10)-tiene-3, $10^{-5} \mathrm{~mol} / \mathrm{l}$; Tocris Bioscience) for $24 \mathrm{~h}$. No intervention was used as a blank control.

Subsequently, the cells were fixed in $4 \%$ paraformaldehyde (Sigma) for $30 \mathrm{~min}$ at room temperature and then permeabilized with $0.25 \%$ Triton X-100 (Sigma) in PBS for $20 \mathrm{~min}$ at $25^{\circ} \mathrm{C}$. The fixed and permeabilized cells were blocked in $1 \%$ BSA and incubated overnight at $4{ }^{\circ} \mathrm{C}$ with a 1:100 dilution of anti-SREBP2 antibody (Abcam). Then the cells were incubated with a 1:200 dilution of Alexa Fluor 594-labelled mouse anti-rabbit IgG (Invitrogen) for $2 \mathrm{~h}$. The nuclei were stained with $0.125 \mu \mathrm{g} / \mathrm{ml}$ DAPI for $15 \mathrm{~min}$.

We analyzed the slides with an Olympus BX51TF fluorescence microscope (Olympus Corporation), with excitation/emission at $530 / 580 \mathrm{~nm}$ for the red fluorescence and $358 / 461 \mathrm{~nm}$ for DAPI. Finally, we analyzed the images using Image-J software from the United States National Institutes of Health (http://rsb.info.nih.gov/ij/). The intensity was calculated using the mean grey value. 
Bioinformatics analysis and chromatin immunoprecipitation (ChIP)

We used regulatory sequence analysis tools (http://rsat-new.ccb.sickkids.ca/) to analyze the sequence of the SREBP2 gene promoter to find high-score ERE-like sequences. HepG2 and HuH-7 cells were treated with $10^{-7} \mathrm{~mol} / \mathrm{l} \mathrm{E}_{2}$ for $24 \mathrm{~h}$ and then cross-linked according to the Millipore EZ-ChIP Assay Kit protocol (Millipore).

Immunoprecipitation was performed with the following antibodies purchased from Millipore: mouse anti-human ER $\alpha$ ChIP antibody, mouse IgG used as the negative control, and mouse anti-human RNA Polymerase II antibody used as the positive control.

SREBP2 was then detected via PCR using 5'-GTCTCCAACTCCTGACCTCAA-3' and 5'-AGTGCCTTGCATACTGCTGTA-3' as the primer sequences. The PCR products were analyzed using agarose electrophoresis and the band was excised from the gel. Finally, the PCR product was sequenced by Invitrogen.

\section{Luciferase reporter assay}

The putative or mutated ERE-like sequences were synthesized by GeneCopoeia and the fragments were digested with restriction endonucleases XhoI and KpnI (Thermo Fisher Scientific), which respectively recognize the sequences $\mathrm{C}^{\wedge} \mathrm{TCGAG}$ and $\mathrm{GGTAC} \mathrm{C}^{\wedge}$. Then, they were ligated into the pGL3-basic plasmid (Promega). After that, we completed construction of the luciferase reporter systems of the SREBP2 promoter: SREBP2-Luciferase (SREBP2-Luc) and SREBP2-Mutation (SREBP2-Mut).

The putative ERE-like sequence was GCATTCGCTCCGAGGCCGCGGGGGGA GGGACCTCACTATGCAAATCTGAGCTGCTGATCGATGACGCGCCATCACCCC ACGCACCGCTTCGCTCGCCCATTGGCTGAGATGAGCCTGGTCCCATTGACAA CAAACAGGGGGGCGCGCGGCCTGGAGGCGGGGCCGCAGGGGGCGCGGG CTGGGGCGGGGGAATCCCGCCCCGCC.

The mutated ERE-like sequence was GCATTCGCTCCGAGGCCGCGGGGGGA GGGACCTCACTATGCAAATCTGAGCTGCTGATCGATGACGCGCCATCACCCC ACGCACCGCTTCGCTCGCCCATTGGCTGAGATGAGCCTCCCAGCATTGACAA CAAACAGGGGGGCGCGCGGCCTGGAGGCGGGGCCGCAGGGGGCGCGGG CTGGGGCGGGGGAATCCCGCCCCGCC.

HepG2 and HuH-7 cells were cultured in 6-well plates for $24 \mathrm{~h}$ in phenol red-free DMEM supplemented with 1\% charcoal/dextran-treated FBS. Using Fugene HP transfection reagent (Roche Applied Science), the luciferase reporter plasmid containing the SREBP2 promoter and the pRL-TK reporter plasmid (cDNA encoding Renilla luciferase; Promega) were co-transfected into the cells. After $24 \mathrm{~h}$ of transfection, $10^{-7} \mathrm{~mol} / \mathrm{l}$ $\mathrm{E}_{2}$ was added for an additional $24 \mathrm{~h}$. Luciferase activities in the cell lysates were measured using the dual-luciferase reporter assay system (Promega) according to the manufacturer's instructions. After normalization to the Renilla luciferase activity, luciferase values were calculated.

\section{Statistical analysis}

The 2-tailed Student's t test was used to evaluate the statistical significance of the difference between two groups. One-way ANOVA and Turkey's post hoc tests were used to evaluate the statistical significance of the difference between more than two groups. 
All statistical analyses were carried out using SPSS 16.0. The results were recorded as the means \pm SEM and were considered significantly different at $p<0.05$.

\section{Results}

Elevated SREBP2 expression, target gene expression and lipid secretion in HepG2 cells after treatment with $E_{2}$

SREBP2 mRNA expression in HepG2 cells showed a dose-dependent increase when treated with $E_{2}$ (Fig. 1a). It was found that $E_{2}$ at $10^{-7} \mathrm{~mol} / \mathrm{l}$ increased the expression of the SREBP2 protein in HepG2 cells compared with that in the blank control and DMSO groups (negative control; Fig. 1b). From the immunofluorescence analysis, the mean grey value of SREBP2 in the $\mathrm{E}_{2}$-treated group was significantly higher than that in the control group (Fig. 1c). Furthermore, the increased protein levels were obviously blocked when the cells were treated with ICI (Fig. 1b, c), which is an estrogen receptoralpha $(E R \alpha)$ antagonist [21], indicating that the estrogen receptor was involved.

Furthermore, $E_{2}$ stimulated lipid secretion in HepG2 cells, as could be seen in the increased TC, TG and LDL-C levels and decreased HDL-C levels (Fig. 2a). Increased mRNA expression of the SREBP2 downstream target gene HMGCR (Fig. 2b) and LDLR (Fig. 2c) were found in HepG2 cells after $\mathrm{E}_{2}\left(10^{-7} \mathrm{~mol} / \mathrm{l}\right)$ treatment.

\section{Bioinformatics analysis of the SREBP2 promoter}

The promoter sequence of the SREBP2 gene was analyzed using regulatory sequence analysis tools to identify the putative EREs. The bioinformatics analysis showed that there were multiple possible ERE-binding regions in the SREBP2 promoter. The highscoring ERE-like sequences were: GGTCCcatTGACA (-88 -76), GATGAcatGGACA $(-1510 \sim-1498)$ and GCACAcctCGGCC $(-1675 \sim-1663)$.

\section{Identification of the predicted putative ERE in the SREBP2 promoter}

ChIP analysis was used to confirm that SREBP2 expression is directly regulated by $E_{2}$ through an ERE. Different primers were used to amplify the high-scoring ERE-like sequences in the promoter of SREBP2. The results show an ERE sequence at $-88 \sim-76$ of the SREBP2 promoter (Fig. 3a). Gel extraction of the PCR product was performed and sequencing revealed that the predicted ERE sequence was contained (Fig. 3b). The results suggest that one fragment containing the putative ERE (GGTCCcatTGACA) was captured using ChIP after treatment with $\mathrm{E}_{2}$.

\section{Function of the putative ERE in the SREBP2 promoter}

We transfected HepG2 and HuH-7 cells with luciferase reporter constructs (SREBP2Luc, SREBP2-Mut or blank control). Luciferase activities were measured after treatment with $E_{2}$ (Fig. 4a) to determine whether the putative ERE plays a functional role in estrogen-dependent transcriptional activation. We found that the construct of the putative ERE (GGTCCcatTGACA) could be activated by $E_{2}$, while cells were unaffected when using the construct of the mutated element (CCCAGcatTGACA; Fig. 4b). Our results suggest that a functional ERE motif exists in the SREBP2 gene promoter, and that the ERE motif is involved in mediating estrogen-dependent SREBP2 expression. 


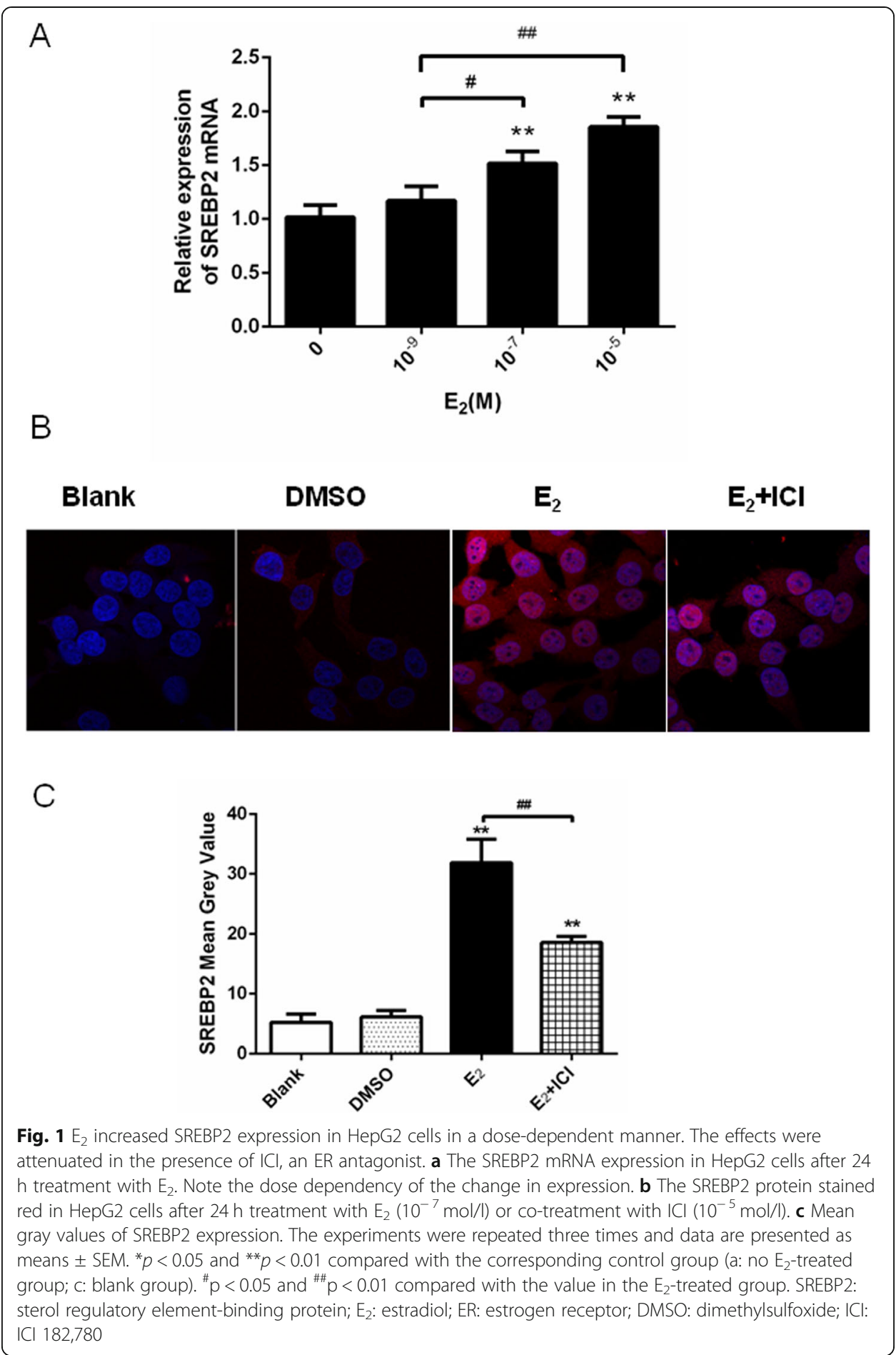

\section{Discussion}

In this study, we found that $E_{2}$ could promote SREBP2 expression in HepG2 cells. Through a bioinformatics analysis of the sequence of the SREBP2 gene promoter, we predicted that there were multiple ERE-like sequences in this region. Furthermore, a functional ERE was identified in the SREBP2 promoter. 


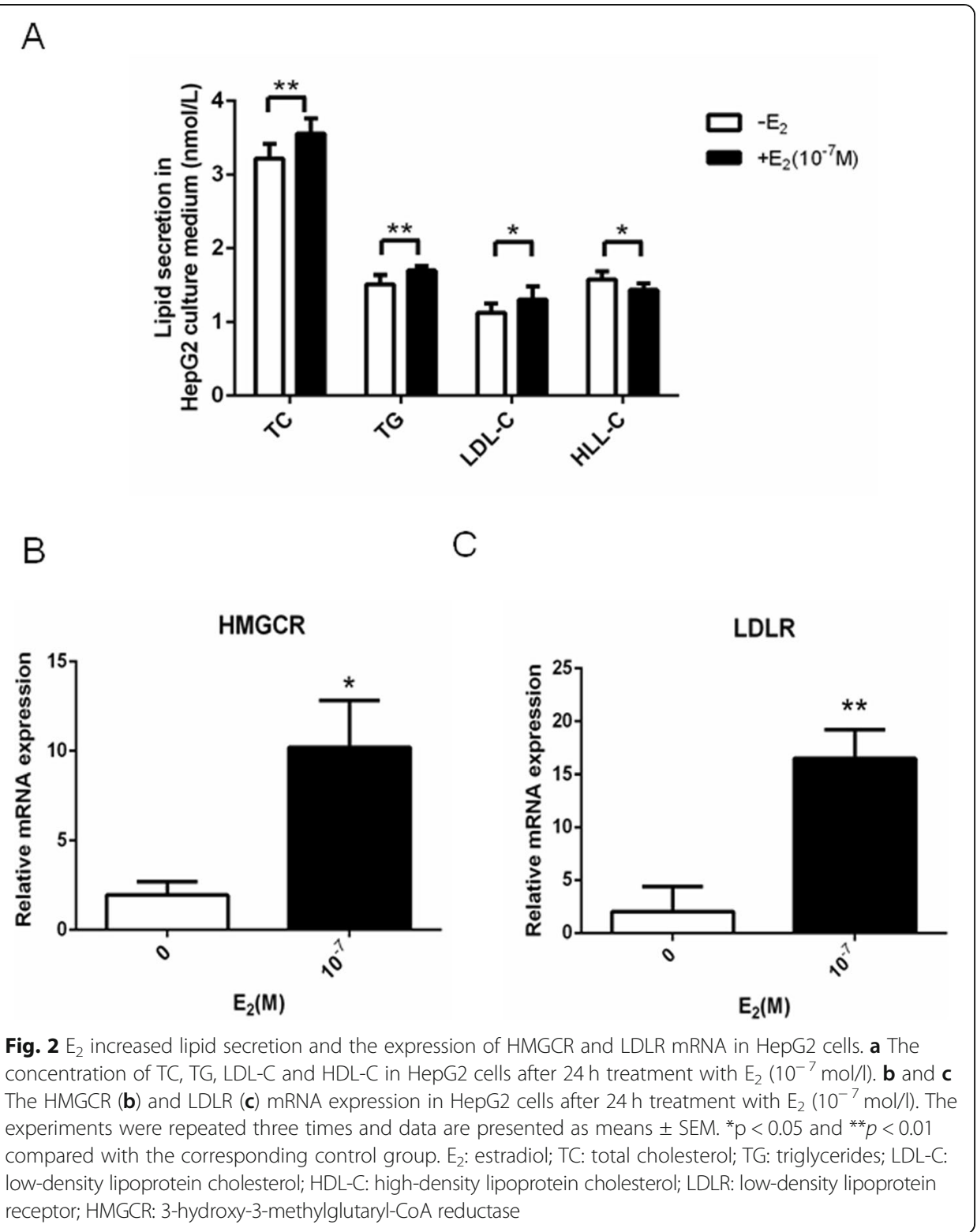

SREBPs belong to a transcription factor superfamily that can activate a series of enzymes involved in lipid biosynthesis and absorption, such as LDLR, HMGCR, HMGCoA synthase and squalene synthase. Therefore, SREBPs play a key role in the lipid metabolic pathway [22, 23]. SREBP1a and SREBP1c are two products of the same gene, located in the $17 \mathrm{p} 11.2$ region $[24,25]$. Produced by another gene located in the 22q13 region, SREBP2 mainly regulates the transcription of enzymes required for cholesterol metabolism [26].

It has been reported that inflammatory cytokines can aggravate cholesterol accumulation in hepatocytes by disturbing SREBP2 regulation, and that SREBP2 expression inhibition can significantly increase cholesterol accumulation [27]. In addition, SREBP2 can specifically bind to SREs on lipid-related genes, such as LDLR and HMGCR, to directly regulate and maintain intracellular cholesterol homeostasis when intracellular cholesterol is deficient $[28,29]$. Thus, SREBP2 expression inhibition could disrupt 
A

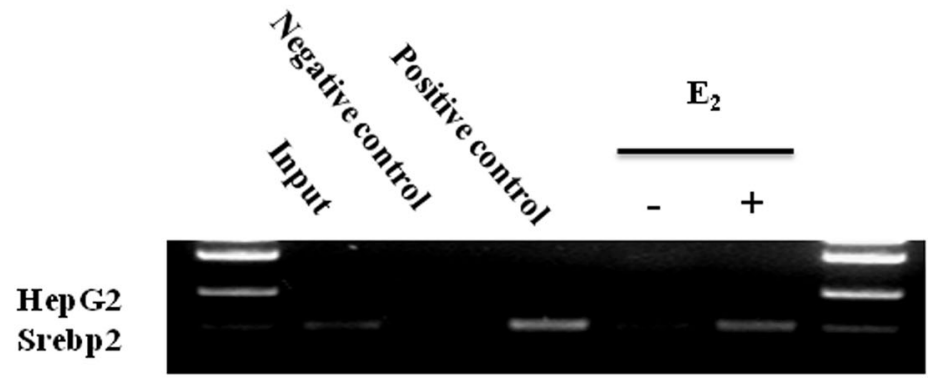

HuH-7

Srebp2

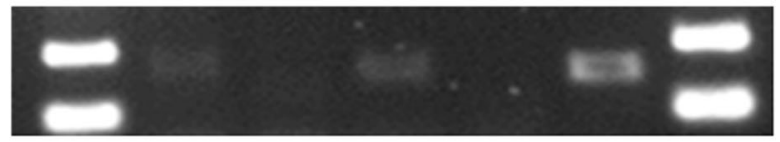

$\mathrm{B}$

GGTCCCATTGACA
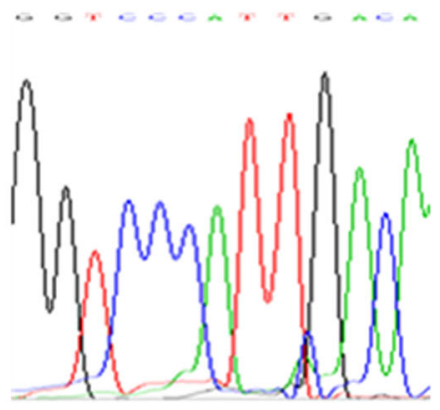

Fig. 3 Existence of an ERE within the SREBP2 promoter. a ChIP analysis was performed using anti-ERa or anti-RNA polymerase II antibody to ascertain the existence of the ERE in the promoter of the SREBP2 gene. The PCR results show that one fragment containing the putative ERE could be precipitated after treatment of HepG2 and HuH-7 with $\mathrm{E}_{2}\left(10^{-7} \mathrm{~mol} / \mathrm{l}\right)$ for $24 \mathrm{~h}$. $\mathbf{b}$ The pulled-down band was excised from the gel and sequenced. SREBP2: sterol regulatory element-binding protein; $E_{2}$ : estradiol; ERE: estrogen response element; ChIP: chromatin immunoprecipitation

SREBP2-mediated LDLR and HMGCR feedback regulation, thereby causing excess accumulation of cholesterol.

Aberrant accumulation of lipids in the arterial wall can lead to atherosclerosis, which plays an important role in the progression of cardiovascular disease [30]. Lipid disorders may easily occur in postmenopausal women due to estrogen deficiency. Low-dose estrogen supplementation can lower the cholesterol level and thus reduce the incidence and mortality of cardiovascular disease [31, 32]. However, the underlying mechanisms still need to be completely elucidated.

In this study, we found that $\mathrm{E}_{2}$ could regulate SREBP2 expression. This was validated by SREBP2 expression inhibition after treatment with the ER $\alpha$ antagonist ICI. The expression of SREBP2 mRNA significantly increased after treatment with $10^{-7} \mathrm{~mol} / \mathrm{l}$ and $10^{-5} \mathrm{~mol} / \mathrm{l} \mathrm{E}_{2}$ compared to $10^{-9} \mathrm{~mol} / \mathrm{l} \mathrm{E}_{2}$, which is in the physiological range for premenopausal women [33]. Furthermore, the lipid secretion content in the supernatant of the hepatic cell lines increased and the expressions of the SREBP2 downstream target genes HMGCR and LDLR mRNA were upregulated after $E_{2}$ treatment in hepatic cell lines. Therefore, our results reveal that $E_{2}$ may 
A

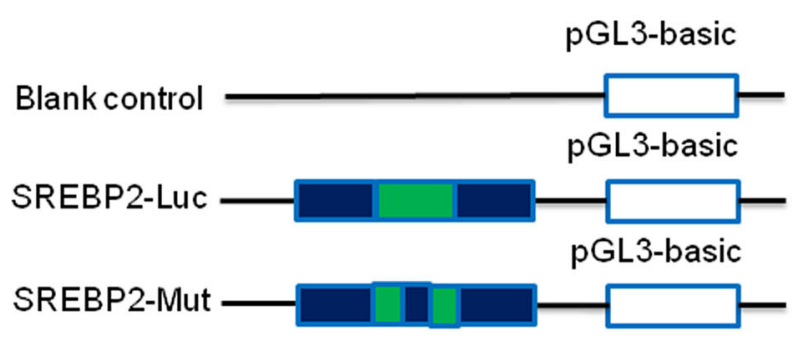

B

HepG2

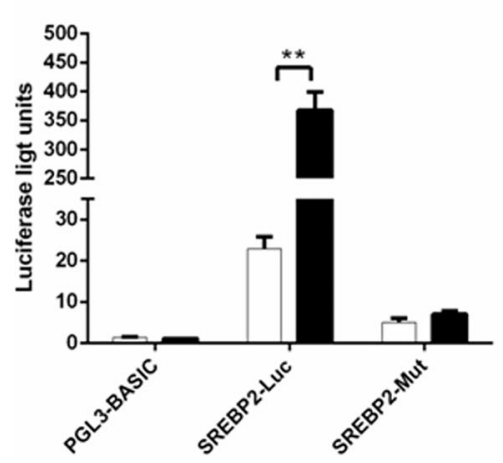

HuH-7

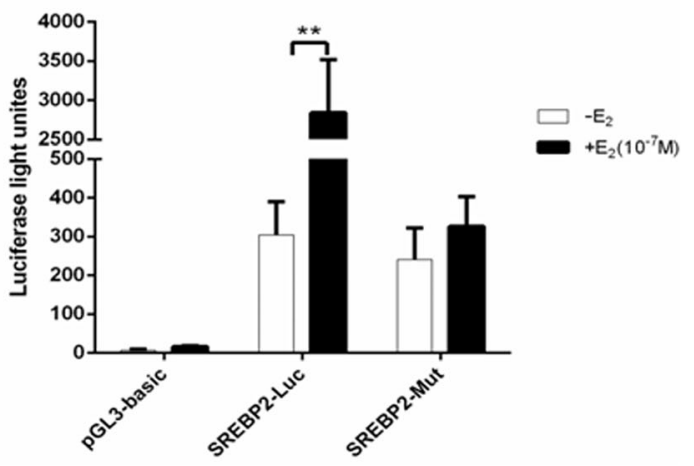

Fig. 4 The ERE was identified functionally within the SREBP2 promoter. a Schematic diagram of luciferase reporter constructs. Blank control: pGL3-basic plasmid; SREBP2-Luc: pGL3-basic plasmid with the putative ERE-like sequence insert; SREBP2-Mut: pGL3-basic plasmid with the mutative ERE-like sequence insert. $\mathbf{b}$ Luciferase activities of three report systems in the presence or absence of $E_{2}\left(10^{-7} \mathrm{~mol} / \mathrm{l}\right)$ were compared with each other. The experiments were repeated three times and data are presented as means \pm SEM. ${ }^{* *} p<0.01$ compared with the value in the non- $E_{2}$-treated control group. SREBP2: sterol regulatory elementbinding protein 2; $E_{2}$ : estradiol

regulate lipid metabolism by affecting SREBP2 expression, and thereby relates to cardiovascular diseases.

Lipids are mainly synthesized in the liver [34], so we used hepatic cell lines to study this mechanism. HepG2 and HuH-7 cells express all three estrogen receptor subtypes: ER $\alpha, E R \beta$ and G protein-coupled estrogen receptor (GPER) [35, 36].

Estrogen can exert multiple-pathway regulatory effects by means of acting on its receptors [37]. Classical estrogen receptors, such as ER $\alpha$ and ER $\beta$, mainly exist in the nucleus and exert their functions through the classical regulatory pathway as follows. Estrogen binds with ER and induces Hsp90 to separate, thus forming ER homologous or heterologous dimers. Activated ER binds with ERE and the ER-ERE complex recruits other proteins to form a transcription initiation complex to induce transcription [12].

There has been evidence of the regulation of SREBP gene transcription as a mechanism to alter SREBP levels. There are binding sites for the thyroid hormone receptor in the 5 '-flanking sequence of SREBP2 [38]. Strikingly, in this study, an ERE site was identified in the SREBP2 gene promoter. Therefore, SREBP2 regulation by estrogen provides a mechanism to link lipid disorder with estrogen. 
We used a luciferase activity assay to analyze the activity of this ERE sequence and found that after $E_{2}$ treatment, the activity of the plasmid containing this ERE sequence was significantly higher than that of the blank control or mutant sequence.

In our study, a functional ERE (GGTCCcatTGACA) was identified in the $-88 \sim-76$ region of the SREBP2 promoter for the first time. The sequence of ERE in the SREBP2 gene promoter is similar to that of typical EREs, which are elements with palindrome structure. The common sequence is 5'-GGTCAnnnTCACC-3' (where $\mathrm{n}$ represents any nucleotide) and contains 13 inverted repeat base pairs and 3 bases that can be randomly replaced. However, in the genes regulated by estrogen, only a few contain the canonical ERE sequence and the majority of them have a variation of the common ERE sequence $[39,40]$. Thus, our study showed that $E_{2}$ could directly regulate SREBP2 expression via an ERE in its promoter.

Our findings demonstrate that estrogen directly activates SREBP2 gene expression. An ERE, one binding site for ER $\alpha$, has been identified in the $5^{\prime}$-flanking sequence of SREBP2. It suggests that estrogen may have an effect on lipid metabolism by regulating the SREBP2 promotor. Our results provide a basis for cardiovascular disease prevention and treatment (Fig. 5, schematic of proposed mechanism).

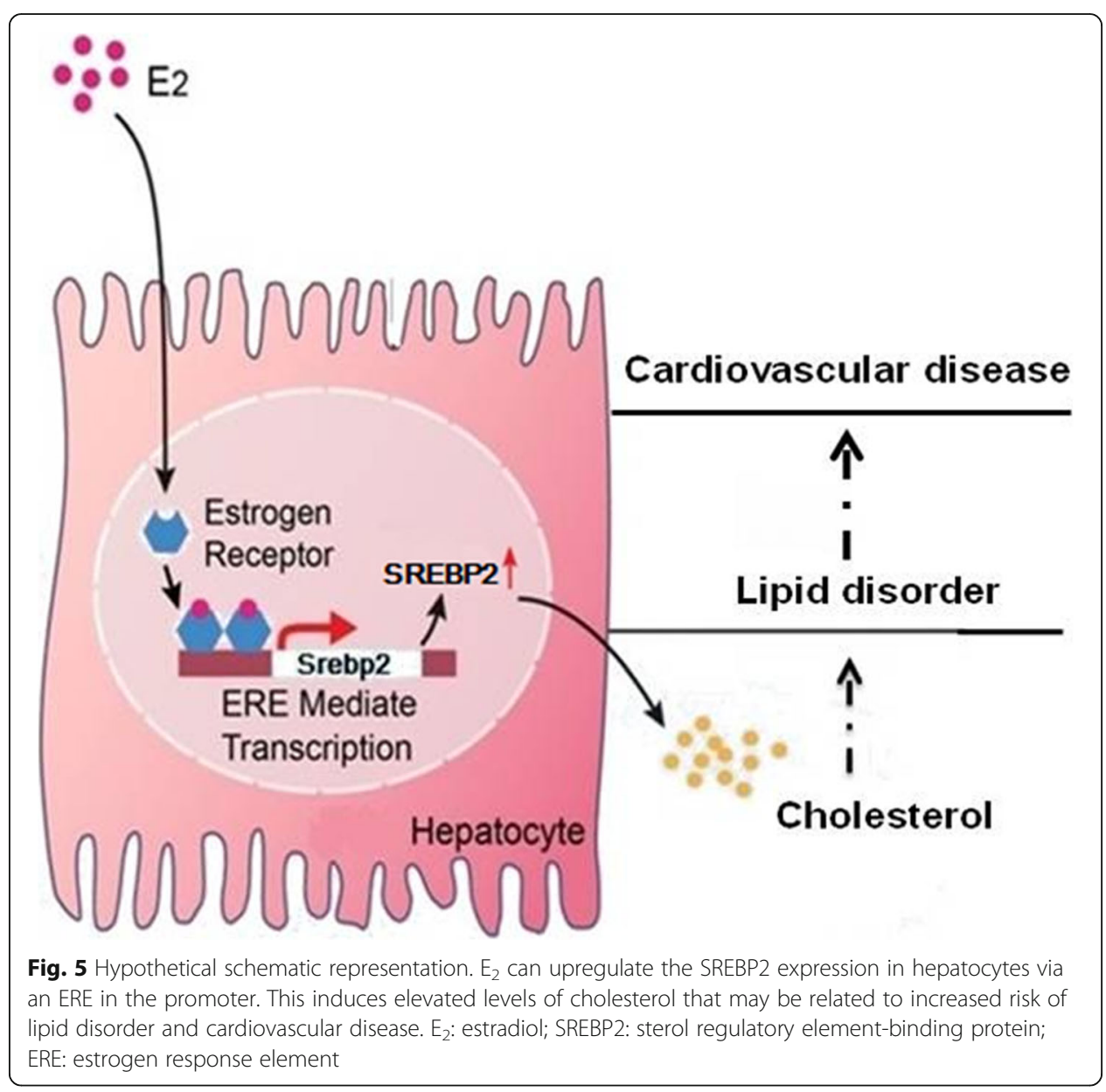




\section{Abbreviations}

ChIP: Chromatin immunoprecipitation; $E_{2}$ : Estradiol; ERE: Estrogen response element; HDL-C: High-density lipoprotein cholestero; HepG2: Human hepatoblastoma cells; HMGCR: 3-hydroxy-3-methylglutaryl-CoA reductase; HuH-7: Human hepatoma cells; LDL-C: Low-density lipoprotein cholesterol; LDLR: Low-density lipoprotein receptor; SREBP2: Sterol regulatory element-binding protein 2; TC: Total cholesterol; TG: triglycerides

\section{Acknowledgements}

Not applicable.

\section{Authors' contributions}

YM designed the study, conducted the experiment and drafted the manuscript. LZ conducted the experiment and statistical analysis. All authors commented on the drafts and approved the final draft.

\section{Funding}

This work was supported by the Natural Science Foundation of China (81801444), Natural Science Foundation of Anhui Province (1808085QH267), and Fundamental Research Funds for the Central Universities (WK9110000049).

\section{Availability of data and materials}

The datasets used and/or analyzed during this study are available from the corresponding author on reasonable request (mengye@ustc.edu.cn).

\section{Ethics approval and consent to participate}

There were no human tissues or animal experiments in this study.

\section{Consent for publication}

Not applicable.

\section{Competing interests}

The authors declare that they have no competing interests.

Received: 27 June 2019 Accepted: 27 November 2019

Published online: 03 December 2019

\section{References}

1. Glovaci D, Fan W, Wong ND. Epidemiology of diabetes mellitus and cardiovascular disease. Curr Cardiol Rep. 2019;21(4):21.

2. Kachur S, Lavie CJ, de Schutter A, Milani RV, Ventura HO. Obesity and cardiovascular diseases. Minerva Med. 2017;108(3): 212-28.

3. Hayashi T, Farrell MA, Chaput LA, Rocha DA, Hernandez M. Lifestyle intervention, behavioral changes, and improvement in cardiovascular risk profiles in the California WISEWOMAN project. J Women's Health (Larchmt). 2010;19(6):1129-38.

4. Koene RJ, Prizment AE, Blaes A, Konety SH. Shared risk factors in cardiovascular disease and Cancer. Circulation. 2016; 133(11):1104-14.

5. Steen DL, Khan I, Becker L, Foody JM, Gorcyca K, Sanchez RJ, et al. Patterns and predictors of lipid-lowering therapy in patients with atherosclerotic cardiovascular disease and/or diabetes mellitus in 2014: insights from a large US managedcare population. Clin Cardiol. 2017;40(3):155-62

6. Banks E, Korda RJ, Stavreski B. Absolute risk of cardiovascular disease events and blood pressure- and lipid-lowering therapy in Australia. Med J Aust. 2017;206(1):51.

7. Piechota-Polanczyk A, Jozkowicz A. The role of statins in the activation of Heme Oxygenase-1 in cardiovascular diseases. Curr Drug Targets. 2017;18(6):674-86.

8. Hasani-Ranjbar S, Ejtahed HS. Statins alone or polypill for primary prevention of cardiovascular diseases. J Diabetes Metab Disord. 2016:15:55.

9. Van Leer P. The risk of cardiovascular disease, fracture, dementia, and Cancer after long-term hormone therapy in Perimenopausal and postmenopausal women. Am Fam Physician. 2018;98(2):117-8.

10. Segarra TJ, Ritchie M, Zehtabchi S. Hormone Therapy for Primary Prevention of Cardiovascular Disease in Postmenopausal Women. Am Fam Physician. 2018;98(7):418B.

11. Cutchins A, Wenger NK. Is there a role for menopausal hormone therapy (MHT) for cardiovascular disease prevention in select postmenopausal women? Curr Treat Options Cardiovasc Med. 2013;15(6):722-34.

12. Klinge CM. Estrogen receptor interaction with estrogen response elements. Nucleic Acids Res. 2001:29(14):2905-19.

13. Pellegrini M, Pallottini V, Marin R, Marino M. Role of the sex hormone estrogen in the prevention of lipid disorder. Curr Med Chem. 2014;21(24):2734-42.

14. Kaygusuz I, Simavli SA, Eser A, Inegol Gumus I, Yuksel S, Iltemir Duvan ZC, et al. Effects of oral hormone replacement therapy on mean platelet volume in postmenopausal women. Turk J Med Sci. 2014;44(6):980-4.

15. Sassarini J, Lumsden MA. Oestrogen replacement in postmenopausal women. Age Ageing. 2015;44(4):551-8.

16. Eberle D, Hegarty B, Bossard P, Ferre P, Foufelle F. SREBP transcription factors: master regulators of lipid homeostasis. Biochimie. 2004:86(11):839-48

17. Mullen E, Brown RM, Osborne TF, Shay NF. Soy isoflavones affect sterol regulatory element binding proteins (SREBPs) and SREBP-regulated genes in HepG2 cells. J Nutr. 2004;134(11):2942-7.

18. Horton JD, Shah NA, Warrington JA, Anderson NN, Park SW, Brown MS, et al. Combined analysis of oligonucleotide microarray data from transgenic and knockout mice identifies direct SREBP target genes. Proc Natl Acad Sci U S A. 2003; 100(21):12027-32. 
19. Bitter A, Nussler AK, Thasler WE, Klein K, Zanger UM, Schwab M, et al. Human sterol regulatory element-binding protein 1a contributes significantly to hepatic lipogenic gene expression. Cell Physiol Biochem. 2015;35(2):803-15.

20. Denver N, Khan S, Homer NZM, MacLean MR, Andrew R. Current strategies for quantification of estrogens in clinical research. J Steroid Biochem Mol Biol. 2019;192:105373.

21. Wakeling AE, Dukes M, Bowler J. A potent specific pure antiestrogen with clinical potential. Cancer Res. 1991;51(15): 3867-73.

22. Miyata S, Inoue J, Shimizu M, Sato R. Xanthohumol improves diet-induced obesity and fatty liver by suppressing sterol regulatory element-binding protein (SREBP) activation. J Biol Chem. 2015;290(33):20565-79.

23. Makdissy N, Haddad K, Mouawad C, Popa I, Younsi M, Valet P, et al. Regulation of SREBPs by Sphingomyelin in adipocytes via a Caveolin and Ras-ERK-MAPK-CREB signaling pathway. PLoS One. 2015;10(7):e0133181.

24. Zhu J, Cui G, Chen M, Xu Q, Wang X, Zhou D, et al. Expression of R132H mutational IDH1 in human U87 glioblastoma cells affects the SREBP1a pathway and induces cellular proliferation. J Mol Neurosci. 2013;50(1):165-71.

25. Gijs HL, Willemarck N, Vanderhoydonc F, Khan NA, Dehairs J, Derua R, et al. Primary cilium suppression by SREBP1C involves distortion of vesicular trafficking by PLA2G3. Mol Biol Cell. 2015;26(12):2321-32.

26. Mastrocola R, Guglielmotto M, Medana C, Catalano MG, Cutrupi S, Borghi R, et al. Dysregulation of SREBP2 induces BACE1 expression. Neurobiol Dis. 2011;44(1):116-24.

27. Zhao L, Chen Y, Tang R, Chen Y, Li Q, Gong J, et al. Inflammatory stress exacerbates hepatic cholesterol accumulation via increasing cholesterol uptake and de novo synthesis. J Gastroenterol Hepatol. 2011;26(5):875-83.

28. Kumar V, Sharma P, Bairagya HR, Sharma S, Singh TP, Tiku PK. Inhibition of human 3-hydroxy-3-methylglutaryl CoA reductase by peptides leading to cholesterol homeostasis through SREBP2 pathway in HepG2 cells. Biochim Biophys Acta, Proteins Proteomics. 2019;1867(6):604-15.

29. Arjuman A, Pandey H, Chandra NC. Effect of a combination oral contraceptive (desogestrel+ethinyl estradiol) on the expression of low-density lipoprotein receptor and its transcription factor (SREBP2) in placental trophoblast cells, Contraception. 2011;84(2):160-8.

30. Ordovas-Montanes JM, Ordovas JM. Cholesterol, Inflammasomes, and Atherogenesis. Curr Cardiovasc Risk Rep. 2012;6(1): 45-52.

31. Rosano GM, Vitale C, Fini M. Cardiovascular aspects of menopausal hormone replacement therapy. Climacteric. 2009; 12(Suppl 1):41-6.

32. Grodstein F, Manson JE, Colditz GA, Willett WC, Speizer FE, Stampfer MJ. A prospective, observational study of postmenopausal hormone therapy and primary prevention of cardiovascular disease. Ann Intern Med. 2000;133(12):933-41.

33. Jordan VC, Fritz NF, Langan-Fahey S, Thompson M, Tormey DC. Alteration of endocrine parameters in premenopausal women with breast cancer during long-term adjuvant therapy with tamoxifen as the single agent. J Natl Cancer Inst. 1991;83(20):1488-91.

34. Jiang J, Nilsson-Ehle P, Xu N. Influence of liver cancer on lipid and lipoprotein metabolism. Lipids Health Dis. 2006;5:4.

35. Shen M, Shi H. Estradiol and estrogen receptor agonists oppose oncogenic actions of Leptin in HepG2 cells. PLoS One. 2016;11(3):e0151455.

36. Hayashida K, Shoji I, Deng L, Jiang DP, Ide YH, Hotta H. 17ß-estradiol inhibits the production of infectious particles of hepatitis C virus. Microbiol Immunol. 2010;54(11):684-90.

37. Meyer MR, Haas E, Prossnitz ER, Barton M. Non-genomic regulation of vascular cell function and growth by estrogen Mol Cell Endocrinol. 2009;308(1-2):9-16.

38. Shin DJ, Osborne TF. Thyroid hormone regulation and cholesterol metabolism are connected through sterol regulatory element-binding Protein-2 (SREBP-2). J Biol Chem. 2003;278(36):34114-8.

39. Hyder SM, Stancel GM. In vitro interaction of uterine estrogen receptor with the estrogen response element present in the 3'-flanking region of the murine c-fos protooncogene. J Steroid Biochem Mol Biol. 1994;48(1):69-79.

40. Hou Y, Zhang S, Wang L, Li J, Qu G, He J, et al. Estrogen regulates iron homeostasis through governing hepatic hepcidin expression via an estrogen response element. Gene. 2012;511(2):398-403.

\section{Publisher's Note}

Springer Nature remains neutral with regard to jurisdictional claims in published maps and institutional affiliations.

\section{Ready to submit your research? Choose BMC and benefit from}

- fast, convenient online submission

- thorough peer review by experienced researchers in your field

- rapid publication on acceptance

- support for research data, including large and complex data types

- gold Open Access which fosters wider collaboration and increased citations

- maximum visibility for your research: over 100M website views per year

At $\mathrm{BMC}$, research is always in progress.

Learn more biomedcentral.com/submissions 